\title{
A FORMAL DERIVATION OF THE ARONSSON EQUATIONS FOR SYMMETRIZED GRADIENTS
}

\author{
MARK SPANIER \\ Department of Mathematics \\ North Dakota State University \\ NDSU Dept. \# 2750, P.O. Box 6050, \\ Fargo, ND 58108-6050 U.S.A. \\ E-mail: mark.spanier@gmail.com
}

\begin{abstract}
The Euler-Lagrange equations associated to the problem of minimizing a power-law functional acting on symmetrized gradients are identified. A formal derivation of the limiting system of partial differential equations stemming from these equations as $p$ tends to infinity is provided. Our computations are reminiscent of the derivation of the infinity Laplace equation starting from the $p$-Dirichlet integral.
\end{abstract}

\section{INTRODUCTION}

The problem of finding, among all functions with prescribed boundary conditions, those which minimize a given functional is central in the study of calculus of variations. There is a close connection between this problem and the study of ordinary or partial differential equations; it turns out that minimizers of certain integral functionals are solutions to the so-called EulerLagrange equations. These equations are differential equations associated to the minimization problem. A well known example in this direction is that given a bounded open domain $\Omega$ in $\mathbb{R}^{N}(N \geq 1)$ with smooth boundary $\partial \Omega$, minimizers $u \in C^{2}(\bar{\Omega})$ of the Dirichlet functional

$$
I(u)=\frac{1}{2} \int_{\Omega}|\nabla u(x)|^{2} d x,
$$

subject to the boundary condition $u=g$ on $\partial \Omega$, where $g: \partial \Omega \rightarrow \mathbb{R}$ is a given continuous function, are solutions of the boundary value problem

$$
\left\{\begin{array}{l}
-\Delta u=0 \text { in } \Omega \\
u=g \text { on } \partial \Omega
\end{array}\right.
$$

where

$$
\Delta u=\sum_{i=1}^{N} \frac{\partial^{2} u}{\partial x_{i}^{2}}
$$

This paper is based on work done under the supervision of Marian Bocea while the author was an undergraduate student at North Dakota State University. 
is the Laplace operator. The converse of the above statement is also true, that is, solutions of (2) are minimizers of $I$ in the admissible class

$$
\mathcal{A}=\left\{u \in C^{2}(\bar{\Omega}): u=g \text { on } \partial \Omega\right\} .
$$

This is called "Dirichlet's Principle" (see, e.g., Evans [12]). Similarly, minimizing the $p$-Dirichlet functional $(p \geq 2)$

$$
I_{p}(u)=\frac{1}{p} \int_{\Omega}|\nabla u(x)|^{p} d x
$$

leads to the $p$-Laplace equation

$$
-\Delta_{p} u:=-\operatorname{div}\left(|\nabla u|^{p-2} \nabla u\right)=0 .
$$

During the recent decades there has been an increasing interest in understanding asymptotic behavior, as $p \rightarrow \infty$ of minimizers of the $p$-Dirichlet functional $I_{p}$ and its generalizations. This is motivated in part by applications to problems arising in materials science (see, e.g. Garroni, Nesi, and Ponsiglione [13], Bocea and Nesi [9], and references therein). It turns out that minimizers $u_{p}$ of $I_{p}$ subject to boundary conditions converge, as $p \rightarrow \infty$, to minimizers of the limiting functional

$$
I_{\infty}(u):=\operatorname{ess}_{\sup } \operatorname{su\Omega }_{x \in}|\nabla u(x)| .
$$

Minimizing in a properly interpreted sense supremal functionals of this type is the central problem in the rapidly emerging area of calculus of variations in $L^{\infty}$. Besides materials science, these issues are motivated by applications to other fields (see the discussion in Barron, Jensen, and Wang [7]).

It was first observed by Aronsson [1-5] that studying the minimization problem for $I_{\infty}$ also leads to a PDE, the infinity-Laplace equation:

$$
-\Delta_{\infty} u:=\sum_{i, j=1}^{N} \frac{\partial u}{\partial x_{i}} \frac{\partial u}{\partial x_{j}} \frac{\partial^{2} u}{\partial x_{i} \partial x_{j}}=0,
$$

which can be regarded as a limiting case of the $p$-Laplace equation (4) as $p \rightarrow \infty$ in the following way. For $p>2$, consider a smooth solution $u$ of (4), which is also a minimizer of (3). Note that, after computations, (4) can be rewritten as

$$
-(p-2)|\nabla u|^{p-4} \Delta_{\infty} u-|\nabla u|^{p-2} \Delta u=0 .
$$

Dividing by $(p-2)|\nabla u|^{p-4}$ in (7) brings about the equation

$$
-\Delta_{\infty} u-\frac{1}{p-2}|\nabla u|^{2} \Delta u=0 .
$$

A keen reader may question whether or not division by $(p-2)|\nabla u|^{p-4}$ is permitted on the set where $|\nabla u|=0$. However, in this case the left hand side of (8) also vanishes on this set. Formally letting $p \rightarrow \infty$ in (8) now leads to the infinity-Laplace equation (6). This computation can be made rigorous (see, e.g., Bhattacharya, DiBenedetto, and Manfredi [8]): if $u_{p}$ is a (weak) solution of (4), then $u_{p}$ converges uniformly on compact subsets 
of $\Omega$ to a "viscosity solution" of (6), called an $\infty$-harmonic function. We recall that a continuous function $u$ is a viscosity solution of (6) if on one hand, for every local maximum point $x \in \Omega$ of $u-\varphi$, where $\varphi$ is a $C^{2}$ function in a neighborhood of $x$ and $u(x)=\varphi(x)$, we have $-\Delta_{\infty} \varphi(x) \leq 0$, and on the other hand, whenever $x \in \Omega$ is a local minimum point of $u-\varphi$, where $\varphi$ is a $C^{2}$ function in a neighborhood of $x$ and $u(x)=\varphi(x)$, we have $-\Delta_{\infty} \varphi(x) \geq 0$. An excellent account on the theory of infinity harmonic functions can be found in Aronsson, Crandall, and Juutinen [6].

In this paper we consider smooth minimizers $u: \Omega \rightarrow \mathbb{R}^{3}$ of functionals of the form

$$
u \mapsto \int_{\Omega}|e(u)|^{p} d x,
$$

acting on symmetrized gradients $e(u)=\frac{1}{2}\left(\nabla u+(\nabla u)^{T}\right)$ of vector-valued maps $u: \Omega \rightarrow \mathbb{R}^{3}$, we identify the Euler-Lagrange equation that such minimizers must solve, and we formally derive the system of PDEs suggested by these equations as $p \rightarrow \infty$. It turns out that the structure of the individual equations in the limiting system is strikingly similar to the $\infty$-Laplace equation (6).

\section{BACKGROUND}

In this section we recall several definitions and results which are needed in the sequel. The first one is an old friend from vector calculus, Green's First Identity.

Lemma 2.1 (Green's First Identity [16]). Let $u$ and $v$ be functions defined on $\Omega \subset \mathbb{R}^{3}$. Assume that $u$ is twice differentiable and that $v$ is differentiable in $\Omega$. Then

$$
\int_{\partial \Omega} v \frac{\partial u}{\partial n} d S=\int_{\Omega} \nabla v \cdot \nabla u d x+\int_{\Omega} v \Delta u d x
$$

where $\frac{\partial u}{\partial n}=\mathbf{n} \cdot \nabla u$ is the directional derivative of $u$ in the outward normal direction.

Definition 2.2 (Support [15]). The support of a continuous function $f$ : $\Omega \rightarrow \mathbb{R}$ is the closure of the set $\{x \in \Omega: f(x) \neq 0\}$. The collection of all real continuous functions on $\Omega$ whose support is compact is denoted by $C_{c}(\Omega)$.

Definition 2.3 (Function Space $C^{n}\left(\bar{\Omega} ; \mathbb{R}^{N}\right)$ ). Let $\Omega \subset \mathbb{R}^{N}$ be an open set, and let $n \in \mathbb{N} \cup\{\infty\}$. The space $C^{n}\left(\bar{\Omega} ; \mathbb{R}^{N}\right)$ is the collection of all functions $f: \Omega \rightarrow \mathbb{R}^{N}, f=\left(f^{1}, f^{2}, \ldots, f^{N}\right)$, such that, for each $i=1, \ldots, N, f^{i} \in C^{n}(\bar{\Omega})$ the space of $n$ times differentiable functions defined on $\bar{\Omega}$.

The next statement is a key result from real analysis which is sometimes called The Fundamental Theorem of Calculus of Variations.

Lemma 2.4 (Du Bois-Reymond Lemma [10]). Let $f: \Omega \rightarrow \mathbb{R}$ be a locally integrable function such that

$$
\int_{\Omega} f(x) \varphi(x) d x=0, \forall \varphi \in C_{c}(\Omega) .
$$


Then $f=0$ almost everywhere in $\Omega$. In particular, if $f$ is continuous in $\Omega$, the conclusion reads: $f=0$ in $\Omega$.

\section{The DeRIVATION OF THE LIMITING EQUATIONS}

Let $p \geq 2$ and let $\Omega \subset \mathbb{R}^{3}$ be an open, bounded domain with smooth boundary. We consider the problem of minimizing the functional

$$
I(u)=\int_{\Omega}\left|\nabla u(x)+\nabla u(x)^{T}\right|^{p} d x
$$

among all possible functions $u \in C^{2}\left(\bar{\Omega} ; \mathbb{R}^{3}\right)$ belonging to the admissible set

$$
\mathcal{A}=\left\{u \in C^{2}\left(\bar{\Omega} ; \mathbb{R}^{3}\right) \mid u=g \text { on } \partial \Omega\right\},
$$

where $g: \bar{\Omega} \rightarrow \mathbb{R}^{3}$ is a given continuous function. In (9), $|\cdot|$ stands for the usual norm in the space $\mathbb{M}^{3 \times 3}$ given by $|A|=\sqrt{\operatorname{tr}\left(A^{T} A\right)}$, where $A^{T}$ is the transpose of the matrix $A$. In what follows, for given matrices $A, B \in \mathbb{M}^{3 \times 3}$, we denote by $A \cdot B$ the scalar product of $A$ and $B$, defined by $A \cdot B=\operatorname{tr}\left(A B^{T}\right)$.

Assume that $u \in \mathcal{A}$ is a minimizer of $I$, that is $I(u)=\min _{v \in \mathcal{A}} I(v)$. We have $I(u) \leq I(u+t \varphi)$ for all $\varphi=\left(\varphi_{1}, \varphi_{2}, \varphi_{3}\right) \in C^{1}\left(\bar{\Omega} ; \mathbb{R}^{3}\right)$, with $\varphi_{i}=0$ on $\partial \Omega$. Thus, we must have $\left.\frac{d}{d t} I(u+t \varphi)\right|_{t=0}=0$. Differentiating

$$
I(u+t \varphi)=\int_{\Omega}\left|\left(\nabla u(x)+\nabla u(x)^{T}\right)+t\left(\nabla \varphi(x)+\nabla \varphi(x)^{T}\right)\right|^{p} d x
$$

with respect to $t$ gives

$$
\begin{aligned}
& \frac{d}{d t} I(u+t \varphi) \\
& =\int_{\Omega} \frac{d}{d t}\left(\left|\nabla u(x)+\nabla u(x)^{T}+t\left(\nabla \varphi(x)+\nabla \varphi(x)^{T}\right)\right|^{2}\right)^{\frac{p}{2}} d x \\
& =\int_{\Omega} \frac{d}{d t}\left(\left|\nabla u(x)+\nabla u(x)^{T}\right|^{2}+t^{2}\left|\nabla \varphi(x)+\nabla \varphi(x)^{T}\right|^{2}\right. \\
& \left.\quad+2 t\left(\nabla u(x)+\nabla u(x)^{T}\right) \cdot\left(\nabla \varphi(x)+\nabla \varphi(x)^{T}\right)\right)^{\frac{p}{2}} d x \\
& =\frac{p}{2} \int_{\Omega}\left(\left|\nabla u(x)+\nabla u(x)^{T}\right|^{2}+t^{2}\left|\nabla \varphi(x)+\nabla \varphi(x)^{T}\right|^{2}\right. \\
& \left.\quad+2 t\left(\nabla u(x)+\nabla u(x)^{T}\right) \cdot\left(\nabla \varphi(x)+\nabla \varphi(x)^{T}\right)\right)^{\frac{p}{2}-1} \\
& \quad\left(2 t\left|\nabla \varphi(x)+\nabla \varphi(x)^{T}\right|^{2}+2\left(\nabla u(x)+\nabla u(x)^{T}\right) \cdot\left(\nabla \varphi(x)+\nabla \varphi(x)^{T}\right)\right) d x .
\end{aligned}
$$


Thus, at $t=0$ we have

$$
\begin{aligned}
& \left.\frac{d}{d t} I(u+t \varphi)\right|_{t=0} \\
& =p \int_{\Omega}\left|\nabla u(x)+\nabla u(x)^{T}\right|^{p-2}\left(\nabla u(x)+\nabla u(x)^{T}\right) \cdot\left(\nabla \varphi(x)+\nabla \varphi(x)^{T}\right) d x,
\end{aligned}
$$

and hence, we must have

$$
\begin{array}{r}
\int_{\Omega}\left|\nabla u(x)+\nabla u(x)^{T}\right|^{p-2}\left(\nabla u(x)+\nabla u(x)^{T}\right) \cdot\left(\nabla \varphi(x)+\nabla \varphi(x)^{T}\right) d x \\
=0 .
\end{array}
$$

In what follows, given any vector function $v=\left(v^{1}, v^{2}, v^{3}\right)$ and $i, j \in\{1,2,3\}$, $v_{x_{i}}^{j}$ will denote the partial derivative of the $j$ th component of $v$ with respect to $x_{i}$. Using this notation we can rewrite $\left(\nabla u(x)+\nabla u(x)^{T}\right) \cdot\left(\nabla \varphi(x)+\nabla \varphi(x)^{T}\right)$ as follows:

$$
\begin{aligned}
(\nabla u(x)+\nabla & \left.u(x)^{T}\right) \cdot\left(\nabla \varphi(x)+\nabla \varphi(x)^{T}\right)= \\
& \left\langle 4 u_{x_{1}}^{1}, 2\left(u_{x_{2}}^{1}+u_{x_{1}}^{2}\right), 2\left(u_{x_{3}}^{1}+u_{x_{1}}^{3}\right)\right\rangle \cdot\left\langle\varphi_{x_{1}}^{1}, \varphi_{x_{2}}^{1}, \varphi_{x_{3}}^{1}\right\rangle+ \\
& \left\langle 2\left(u_{x_{2}}^{1}+u_{x_{1}}^{2}\right), 4 u_{x_{2}}^{2}, 2\left(u_{x_{2}}^{3}+u_{x_{3}}^{2}\right)\right\rangle \cdot\left\langle\varphi_{x_{1}}^{2}, \varphi_{x_{2}}^{2}, \varphi_{x_{3}}^{2}\right\rangle+ \\
& \left.\left\langle 2\left(u_{x_{3}}^{1}+u_{x_{1}}^{3}\right), 2\left(u_{x_{2}}^{3}+u_{x_{3}}^{2}\right), 4 u_{x_{3}}^{3}\right)\right\rangle \cdot\left\langle\varphi_{x_{1}}^{3}, \varphi_{x_{2}}^{3}, \varphi_{x_{3}}^{3}\right\rangle
\end{aligned}
$$

This, with $e(u):=\frac{1}{2}\left(\nabla u(x)+\nabla u(x)^{T}\right),(11)$ becomes

$$
\begin{aligned}
\int_{\Omega}|e(u)|^{p-2} & {\left[\left\langle 2 u_{x_{1}}^{1}, u_{x_{2}}^{1}+u_{x_{1}}^{2}, u_{x_{3}}^{1}+u_{x_{1}}^{3}\right\rangle \cdot \nabla \varphi^{1}\right.} \\
& +\left\langle u_{x_{2}}^{1}+u_{x_{1}}^{2}, 2 u_{x_{2}}^{2}, u_{x_{2}}^{3}+u_{x_{3}}^{2}\right\rangle \cdot \nabla \varphi^{2} \\
& \left.+\left\langle u_{x_{3}}^{1}+u_{x_{1}}^{3}, u_{x_{2}}^{3}+u_{x_{3}}^{2}, 2 u_{x_{3}}^{3}\right\rangle \cdot \nabla \varphi^{3}\right] d x=0 .
\end{aligned}
$$

Taking $\varphi=\left(\varphi^{1}, 0,0\right),\left(0, \varphi^{2}, 0\right)$, and $\left(0,0, \varphi^{3}\right)$, with $\varphi^{i} \in C_{c}^{\infty}(\Omega), i \in$ $\{1,2,3\}$, we obtain:

$$
\begin{aligned}
& \int_{\Omega}|e(u)|^{p-2}\left\langle 2 u_{x_{1}}^{1}, u_{x_{2}}^{1}+u_{x_{1}}^{2}, u_{x_{3}}^{1}+u_{x_{1}}^{3}\right\rangle \cdot \nabla \varphi^{1} d x=0, \text { for all } \varphi^{1} \in C_{c}^{\infty}(\Omega) ; \\
& \int_{\Omega}|e(u)|^{p-2}\left\langle u_{x_{2}}^{1}+u_{x_{1}}^{2}, 2 u_{x_{2}}^{2}, u_{x_{2}}^{3}+u_{x_{3}}^{2}\right\rangle \cdot \nabla \varphi^{2} d x=0 \text {, for all } \varphi^{2} \in C_{c}^{\infty}(\Omega) ; \\
& \int_{\Omega}|e(u)|^{p-2}\left\langle u_{x_{3}}^{1}+u_{x_{1}}^{3}, u_{x_{2}}^{3}+u_{x_{3}}^{2}, 2 u_{x_{3}}^{3}\right\rangle \cdot \nabla \varphi^{3} d x=0, \text { for all } \varphi^{3} \in C_{c}^{\infty}(\Omega) .
\end{aligned}
$$

In view of Lemmas 2.1 and 2.4 we obtain that $u=\left(u^{1}, u^{2}, u^{3}\right)$ must satisfy the following system of PDEs:

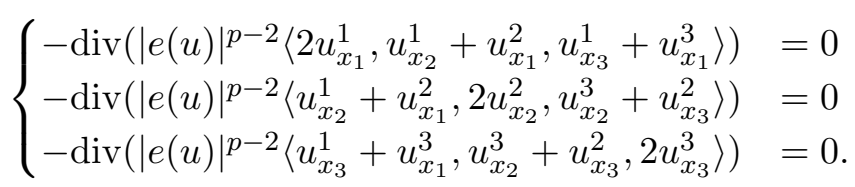


This can be written as

$$
-\operatorname{Div}\left(|e(u)|^{p-2} e(u)\right)=0,
$$

where, for smooth matrix-valued maps $U: \Omega \rightarrow \mathbb{M}^{3 \times 3}$ the differential operator Div is defined by

$$
\operatorname{Div} U:=\left(\begin{array}{c}
\operatorname{div} U^{(1)} \\
\operatorname{div} U^{(2)} \\
\operatorname{div} U^{(3)}
\end{array}\right),
$$

with $U^{(i)}(x):=\left(U_{i 1}(x), U_{i 2}(x), U_{i 3}(x)\right), i=1,2,3$, standing for the $i^{\text {th }}$ row of the matrix $U(x), x \in \Omega$. Note the similarities between (13) and the $p$-Laplace equation (4) which one obtains in the scalar case starting from the $p$-Dirichlet integral. By definition of the divergence operator the first equation in (12) reads

$\frac{\partial}{\partial x_{1}}\left(|e(u)|^{p-2} 2 u_{x_{1}}^{1}\right)+\frac{\partial}{\partial x_{2}}\left(|e(u)|^{p-2}\left(u_{x_{2}}^{1}+u_{x_{1}}^{2}\right)\right)+\frac{\partial}{\partial x_{3}}\left(|e(u)|^{p-2}\left(u_{x_{3}}^{1}+u_{x_{1}}^{3}\right)\right)=0$.

We have

$$
\begin{aligned}
& \frac{\partial}{\partial x_{k}}|e(u)|^{p-2} \\
& =\frac{\partial}{\partial x_{k}}\left(\sum_{i, j=1}^{3}\left(u_{x_{j}}^{i}+u_{x_{i}}^{j}\right)^{2}\right)^{\frac{p-2}{2}} \\
& =\frac{p-2}{2}\left(\sum_{i, j=1}^{3}\left(u_{x_{j}}^{i}+u_{x_{i}}^{j}\right)^{2}\right)^{\left(\frac{p-2}{2}-1\right)} \frac{\partial}{\partial x_{k}}\left(\sum_{i, j=1}^{3}\left(u_{x_{j}}^{i}+u_{x_{i}}^{j}\right)^{2}\right) \\
& =\frac{p-2}{2}|e(u)|^{p-4} \frac{\partial}{\partial x_{k}}\left(\sum_{i, j=1}^{3}\left(u_{x_{j}}^{i}+u_{x_{i}}^{j}\right)^{2}\right) \\
& =(p-2)|e(u)|^{p-4}\left(\sum_{i, j=1}^{3}\left(u_{x_{j}}^{i}+u_{x_{i}}^{j}\right)\left(u_{x_{j} x_{k}}^{i}+u_{x_{i} x_{k}}^{j}\right)\right) \\
& =(p-2)|e(u)|^{p-4} \delta_{k} u,
\end{aligned}
$$

where

$$
\delta_{k} u:=\sum_{i, j=1}^{3}\left(u_{x_{j}}^{i}+u_{x_{i}}^{j}\right)\left(u_{x_{j} x_{k}}^{i}+u_{x_{i} x_{k}}^{j}\right) .
$$

Using this, the first equation in (12) now becomes

$$
\begin{aligned}
& |e(u)|^{p-2}\left(2 u_{x_{1} x_{1}}^{1}+u_{x_{2} x_{2}}^{1}+u_{x_{3} x_{3}}^{1}+u_{x_{1} x_{2}}^{2}+u_{x_{1} x_{3}}^{3}\right) \\
& \quad+(p-2)|e(u)|^{p-4}\left[2 u_{x_{1}}^{1} \delta_{1} u+\left(u_{x_{2}}^{1}+u_{x_{1}}^{2}\right) \delta_{2} u+\left(u_{x_{3}}^{1}+u_{x_{1}}^{3}\right) \delta_{3} u\right]=0 .
\end{aligned}
$$

After dividing this equation by $(p-2)|e(u)|^{p-4}$ we arrive at 


$$
\begin{aligned}
& \frac{1}{p-2}|e(u)|^{2}\left(2 u_{x_{1} x_{1}}^{1}+u_{x_{2} x_{2}}^{1}+u_{x_{3} x_{3}}^{1}+u_{x_{1} x_{2}}^{2}+u_{x_{1} x_{3}}^{3}\right) \\
& +\left[2 u_{x_{1}}^{1} \delta_{1} u+\left(u_{x_{2}}^{1}+u_{x_{1}}^{2}\right) \delta_{2} u+\left(u_{x_{3}}^{1}+u_{x_{1}}^{3}\right) \delta_{3} u\right]=0
\end{aligned}
$$

which, after formally letting $p \rightarrow \infty$, leads to the limiting equation

$$
2 u_{x_{1}}^{1} \delta_{1} u+\left(u_{x_{2}}^{1}+u_{x_{1}}^{2}\right) \delta_{2} u+\left(u_{x_{3}}^{1}+u_{x_{1}}^{3}\right) \delta_{3} u=0 .
$$

In a similar fashion the last two equations in (12) lead, as $p \rightarrow \infty$, to

$$
\left(u_{x_{1}}^{2}+u_{x_{2}}^{1}\right) \delta_{1} u+2 u_{x_{2}}^{2} \delta_{2} u+\left(u_{x_{3}}^{2}+u_{x_{2}}^{3}\right) \delta_{3} u=0,
$$

and

$$
\left(u_{x_{3}}^{1}+u_{x_{1}}^{3}\right) \delta_{1} u+\left(u_{x_{3}}^{2}+u_{x_{2}}^{3}\right) \delta_{2} u+2 u_{x_{3}}^{3} \delta_{3} u=0,
$$

respectively. In vector notation, (14), (15), and (16) can be collectively rewritten in the form

$$
e(u)\left(\begin{array}{c}
\delta_{1} u \\
\delta_{2} u \\
\delta_{3} u
\end{array}\right)=0 .
$$

We would like to point out that the structure of the PDEs in the system (17) closely resembles that of the $\infty$-Laplace equation (6) which is the corresponding limiting equation in the case of gradients of (scalar) functions. Indeed, first note that (6) can be rewritten as

$$
-\left\langle\nabla\left(|\nabla u|^{2}\right), \nabla u\right\rangle=0,
$$

where $\langle\cdot, \cdot\rangle$ stands for the usual inner product in $\mathbb{R}^{3}$. Next, observe that $2 \delta_{k} u$ is in fact the $k^{\text {th }}$ component of the vector function $\nabla\left(|e(u)|^{2}\right)$. Thus, if we denote by $[e(u)]_{k}$ the $k^{\text {th }}$ row of the matrix-valued function $e(u)$, the system (14)-(16) or, equivalently, (17), may be rewritten as

$$
\left\{\begin{array}{l}
-\left\langle\nabla\left(|e(u)|^{2}\right),[e(u)]_{1}\right\rangle=0 \\
-\left\langle\nabla\left(|e(u)|^{2}\right),[e(u)]_{2}\right\rangle=0 \\
-\left\langle\nabla\left(|e(u)|^{2}\right),[e(u)]_{3}\right\rangle=0
\end{array}\right.
$$

Given the rich literature available regarding the existence, uniqueness and regularity of viscosity solutions for the $\infty$-Laplace equation, it would be interesting to pursue similar questions for solutions, in a suitable weak sense, of the limiting system (19) derived in this paper. However, it is still not clear what the right notion of solution should be in this case. Although various definitions of viscosity solutions for systems of PDEs have been introduced in the literature (see, e.g., [11], [14]), we have not yet been able to identify one which is applicable to the special structure of (19).

Acknowledgements. The author would like to thank Dr. Marian Bocea for his thoughtful insight and guidance in this project. 


\section{MARK SPANIER}

\section{REFERENCES}

[1] G. Aronsson. Minimization problems for the functional $\sup _{x} F\left(x, f(x), f^{\prime}(x)\right)$. Ark. Mat., 6:33-53, 1965.

[2] G. Aronsson. Minimization problems for the functional $\sup _{x} F\left(x, f(x), f^{\prime}(x)\right)$. II. Ark. Mat., 6:409-431, 1966.

[3] G. Aronsson. Extension of functions satisfying Lipschitz conditions. Ark. Mat., 6:551561, 1967.

[4] G. Aronsson. On the partial differential equation $u_{x}{ }^{2} u_{x x}+2 u_{x} u_{y} u_{x y}+u_{y}^{2} u_{y y}=0$. Ark. Mat., 7:395-425, 1968.

[5] G. Aronsson. Minimization problems for the functional $\sup _{x} F\left(x, f(x), f^{\prime}(x)\right)$. III. Ark. Mat., 7:509-512, 1969.

[6] G. Aronsson, M. G. Crandall, and P. Juutinen. A tour of the theory of absolutely minimizing functions. Bull. Amer. Math. Soc. (N.S.), 41(4):439-505 (electronic), 2004.

[7] E. N. Barron, R. R. Jensen, and C. Y. Wang. The Euler equation and absolute minimizers of $L^{\infty}$ functionals. Arch. Ration. Mech. Anal., 157(4):255-283, 2001.

[8] T. Bhattacharya, E. DiBenedetto, and J. Manfredi. Limits as $p \rightarrow \infty$ of $\Delta_{p} u_{p}=f$ and related extremal problems. Rend. Sem. Mat. Univ. Politec. Torino, (Special Issue):1568 (1991), 1989. Some topics in nonlinear PDEs (Turin, 1989).

[9] M. Bocea and V. Nesi. $\Gamma$-convergence of power-law functionals, variational principles in $L^{\infty}$, and applications. SIAM J. Math. Anal., 39(5):1550-1576, 2008.

[10] H. Brezis. Analyse fonctionnelle. Théorie et applications. Collection Mathématiques Appliquées pour la Maîtrise. Masson, Paris, 1983.

[11] H. Engler and S. M. Lenhart. Viscosity solutions for weakly coupled systems of Hamilton-Jacobi equations. Proc. London Math. Soc. (3), 63(1):212-240, 1991.

[12] L. C. Evans. Partial differential equations, volume 19 of Graduate Studies in Mathematics. American Mathematical Society, Providence, RI, 1998.

[13] A. Garroni, V. Nesi, and M. Ponsiglione. Dielectric breakdown: optimal bounds. $R$. Soc. Lond. Proc. Ser. A Math. Phys. Eng. Sci., 457(2014):2317-2335, 2001.

[14] H. Ishii and S. Koike. Viscosity solutions for monotone systems of second-order elliptic PDEs. Comm. Partial Differential Equations, 16(6-7):1095-1128, 1991.

[15] W. Rudin. Real and complex analysis. McGraw-Hill Book Co., New York, third edition, 1987.

[16] W. A. Strauss. Partial differential equations. An Introduction. John Wiley \& Sons Ltd., second edition, 2008. 